\title{
Dyslipidemia in patients with chronic kidney disease: etiology and management
}

\author{
Ivana Mikolasevic ${ }^{1,2}$ \\ Marta Žutelija ${ }^{3}$ \\ Vojko Mavrinac' \\ Lidija Orlic ${ }^{2}$ \\ 'Department of Gastroenterology, \\ 2Department of Nephrology, Dialysis \\ and Kidney Transplantation, UHC \\ Rijeka, ${ }^{3}$ School of Medicine, Rijeka, \\ Croatia
}

This article was published in the following Dove Press journal: International Journal of Nephrology and Renovascular Disease 7 February 2017

Number of times this article has been viewed
Correspondence: Ivana Mikolasevic Department of Nephrology, Dialysis and Kidney Transplantation, Department of Gastroenterology, UHC Rijeka, Kresimirova 42, Rijeka 51000, Croatia Tel +385 51658 I22

Email ivana.mikolasevic@gmail.com

\begin{abstract}
Patients with chronic kidney disease (CKD), including those with end-stage renal disease, treated with dialysis, or renal transplant recipients have an increased risk for cardiovascular disease (CVD) morbidity and mortality. Dyslipidemia, often present in this patient population, is an important risk factor for CVD development. Specific quantitative and qualitative changes are seen at different stages of renal impairment and are associated with the degree of glomerular filtration rate declining. Patients with non-dialysis-dependent CKD have low high-density lipoproteins (HDL), normal or low total cholesterol (TC) and low-density lipoprotein (LDL) cholesterol, increased triglycerides as well as increased apolipoprotein B (apoB), lipoprotein(a) (Lp (a)), intermediate- and very-low-density lipoprotein (IDL, VLDL; "remnant particles"), and small dense LDL particles. In patients with nephrotic syndrome lipid profile is more atherogenic with increased TC, LDL, and triglycerides. Lipid profile in hemodialysis (HD) patients is usually similar to that in non-dialysis-dependent CKD patients. Patients on peritoneal dialysis (PD) have more altered dyslipidemia compared to HD patients, which is more atherogenic in nature. These differences may be attributed to PD per se but may also be associated with the selection of dialytic modality. In renal transplant recipients, TC, LDL, VLDL, and triglycerides are elevated, whereas HDL is significantly reduced. Many factors can influence post-transplant dyslipidemia including immunosuppressive agents. This patient population is obviously at high risk; hence, prompt diagnosis and management are required to improve their clinical outcomes. Various studies have shown statins to be effective in the cardiovascular risk reduction in patients with mild-to-moderate $\mathrm{CKD}$ as well as in renal transplant recipients. However, according to recent clinical randomized controlled trials (4D, A Study to Evaluate the Use of Rosuvastatin in Subjects on Regular Dialysis: an Assessment of Survival and Cardiovascular Events, and Study of Heart and Renal protection), these beneficial effects are uncertain in dialyzed patients. Therefore, further research for the most suitable treatment options is needed.
\end{abstract}

Keywords: chronic kidney disease, cardiovascular disease, dyslipidemia

\section{Introduction}

Chronic kidney disease (CKD) is one of today's leading public health problems, with increasing frequency and prevalence. According to the Kidney Disease Outcomes Quality Initiative (K/DOQI), CKD is defined as kidney damage or a decreased kidney glomerular filtration rate (GFR) of $<60 \mathrm{~mL} / \mathrm{min} / 1.73 \mathrm{~m}^{2}$ for at least 3 months. ${ }^{1,2}$ Patients with CKD, particularly those with end-stage renal disease (ESRD) who are treated with hemodialysis (HD) or peritoneal dialysis (PD) or those treated with renal transplantation, are at increased risk of developing cardiovascular disease (CVD). ${ }^{3}$ It is more likely that patients with CKD will die because of CVD than develop ESRD and have the need for renal replacement therapy. ${ }^{3,4}$ Compared with the general population, patients with $\mathrm{CKD}$ 
have an increased risk for developing CVD due to several risk factors often associated with CKD. ${ }^{5}$ These risk factors can be both traditional ones such as age, male gender, diabetes, obesity, hypertension, and dyslipidemia and non-traditional uremia-related risk factors such as anemia, hyperhomocysteinemia, mineral bone disease-CKD with hyperparathyroidism, oxidative stress, hypoalbuminemia, and chronic inflammation. ${ }^{3,4}$ In the general population, high total cholesterol (TC), high low-density lipoprotein (LDL) cholesterol, high triglyceride, and low high-density lipoprotein (HDL) cholesterol are all well-established risk factors for CVD development. However, CKD, with both dialysis and transplantation, is associated with specific qualitative and quantitative lipid abnormalities, resulting in specific dyslipidemia (Table 1). ${ }^{1,3,6}$ The relationship between dyslipidemia and CVD morbidity and mortality in this specific population group is not as clear as that in the general population. ${ }^{7}$ Specific abnormalities in the lipoprotein metabolism, caused by an inappropriate activity of some key enzymes and metabolic pathways, develop in the early stage of renal failure and result in dyslipidemia which presents a risk factor for the atherosclerosis development. Along with CKD progression, metabolic abnormalities may progress further, contributing to atherosclerotic changes and adversely affecting renal function. ${ }^{1,4,7}$ According to the current evidence, lipid-lowering medications are effective in reducing CVD mortality and morbidity in the general population. Recent studies suggest that these medications may have the

Table I Trend of changes in lipids, lipoproteins, and apoA-IV in various stages of $C K D^{6}$

\begin{tabular}{lllll}
\hline Parameter & CKD & $\begin{array}{l}\text { Nephrotic } \\
\text { syndrome }\end{array}$ & Hemodialysis & $\begin{array}{l}\text { Peritoneal } \\
\text { dialysis }\end{array}$ \\
\hline Total cholesterol & $\nearrow$ & $\uparrow \uparrow$ & $\leftrightarrow \downarrow$ & $\uparrow$ \\
LDL cholesterol & $\nearrow$ & $\uparrow \uparrow$ & $\leftrightarrow \downarrow$ & $\uparrow$ \\
HDL cholesterol & $\downarrow$ & $\downarrow$ & $\downarrow$ & $\downarrow$ \\
Non-HDL & $\nearrow$ & $\uparrow \uparrow$ & $\leftrightarrow \downarrow$ & $\uparrow$ \\
cholesterol & & & & \\
TG & $\nearrow$ & $\uparrow \uparrow$ & $\uparrow$ & $\uparrow$ \\
LP (a) & $\nearrow$ & $\uparrow \uparrow$ & $\uparrow$ & $\uparrow \uparrow$ \\
ApoA-I & $\searrow$ & $\nearrow$ & $\downarrow$ & $\downarrow$ \\
ApoA-IV & $\nearrow$ & $\uparrow У$ & $\uparrow$ & $\uparrow$ \\
ApoB & $\nearrow$ & $\uparrow \uparrow$ & $\leftrightarrow \downarrow$ & $\uparrow$
\end{tabular}

Notes: Non-HDL cholesterol includes cholesterol in LDL, VLDL, IDL, and chylomicron and its remnant. Explanation of arrows: normal $(\leftrightarrow)$, increased $(\uparrow)$, markedly increased $(\uparrow \uparrow)$, and decreased $(\downarrow)$ plasma levels compared with nonuremic individuals; increasing $(\nearrow)$ and decreasing $(\searrow)$ plasma levels with decreasing GFR. Copyright @2007 American Society of Nephrology. Kwan BCH, Kronenberg F, Beddhu S, Cheung AK. Lipoprotein Metabolism and lipid management in chronic kidney disease. J Am Soc Nephrol. 2007; 18:1246-1261.6

Abbreviations: apoA-IV, apolipoprotein A-IV; CKD, chronic kidney disease; LDL, low-density lipoprotein; HDL, high-density lipoprotein; TG, triglyceride; LP (a), lipoprotein(a); ApoA-I, apolipoprotein A-I; ApoB, apolipoprotein B; VLDL, verylow-density lipoprotein; IDL, intermediate-density lipoprotein; GFR, glomerular filtration rate. same beneficial effect in both CKD and the transplant population. $^{3}$ This review presents the etiology and pathogenesis of specific lipid abnormalities in patients with CKD stage 1-4 (G1-4), those with nephrotic syndrome, as well as those treated with dialysis and kidney transplantation and ends by summarizing the current evidence and guidelines for the management of dyslipidemia.

\section{Etiology and pathogenesis of dyslipidemia Dyslipidemia in CKD (GI-4)}

Dyslipidemia is a common complication of CKD and lipoprotein metabolism alteration and is associated with the decline in GFR; hence, lipid profile depends on the level of kidney function and the degree of proteinuria (Table 2). ${ }^{8,9}$

Patients with non-dialysis-dependent CKD and without nephrotic syndrome have low HDL and high triglycerides and normal or even low TC and LDL cholesterol, but more atherogenic profile is hidden behind this spectrum. This profile includes increased apolipoprotein B (apoB), lipoprotein (a) (Lp (a)), intermediate- and very-low-density lipoprotein (IDL cholesterol, VLDL cholesterol; "remnant particles"), and small dense LDL particles. Also, in patients with more severe CKD, LDL, and HDL particles are often modified by the oxidative process, that leads into the formation of small lipoproteins and increased formation of oxidized LDL. ${ }^{8,10,11}$

Patients with CKD usually have hypertriglyceridemia due to an increased concentration of triglyceride-rich lipoproteins (VLDL, chylomicrons, and their remnants). Hypertriglyceridemia occurs because of both the delayed catabolism and the increased hepatic production of triglyceride-rich lipoproteins. Delayed catabolism is the most prevalent mechanism responsible for an elevated triglyceride-rich lipoprotein concentration in CKD patients and occurs probably because of a decreased activity of hepatic triglyceride lipase and peripheral lipoprotein lipase. Also, the presence of lipase inhibitors may contribute to delayed triglyceride-rich lipoprotein catabolism. Apolipoprotein C-III (apoC-III) is a direct lipoprotein lipase inhibitor, and its levels are elevated in uremia which further contributes to hypertriglyceridemia. It is also possible that secondary hyperparathyroidism plays an additional role in triglyceride-rich lipoprotein catabolism impairment, resulting in raised plasma triglyceride concentrations associated with CKD. Besides low catabolic activity, increased hepatic production of triglyceride-rich lipoproteins contributes to increased levels of triglycerides in CKD patients. Insulin resistance, often associated with CKD, seems to be responsible for a hepatic VLDL overproduction. ${ }^{10,12}$ 
Table 2 Lipid abnormalities by target population (approximate percentage) ${ }^{9}$

\begin{tabular}{|c|c|c|c|c|}
\hline & $\begin{array}{l}\text { Total cholesterol } \\
>240 \mathrm{mg} / \mathrm{dL}\end{array}$ & $\begin{array}{l}\text { LDL cholesterol } \\
>130 \mathrm{mg} / \mathrm{dL}\end{array}$ & $\begin{array}{l}\text { HDL cholesterol } \\
<35 \mathrm{mg} / \mathrm{dL}\end{array}$ & $\begin{array}{l}\text { Triglyceride } \\
>200 \mathrm{mg} / \mathrm{dL}\end{array}$ \\
\hline General population* & 20 & 40 & 15 & 15 \\
\hline \multicolumn{5}{|l|}{ CKD stages $1-4^{\#}$} \\
\hline With nephrotic syndrome $e^{\ddagger}$ & 90 & 85 & 50 & 60 \\
\hline Without nephrotic syndrome ${ }^{\ddagger}$ & 30 & 10 & 35 & 40 \\
\hline \multicolumn{5}{|l|}{ CKD stage 5} \\
\hline Hemodialysis & 20 & 30 & 50 & 45 \\
\hline Peritoneal dialysis & 25 & 45 & 20 & 50 \\
\hline
\end{tabular}

Notes: *Data from National Health and Nutrition Examination Survey III and the Framingham Offspring Study. ${ }^{32,33}$ \#Data extracted from multiple observational studies of Kasiske. ${ }^{34}$ ₹Nephrotic proteinuria was defined as $>3 \mathrm{~g}$ of total protein excretion in 24 h. Copyright @2004 Society of General Internal Medicine. Weiner DE, Sarnak MJ. Managing dyslipidemia in chronic kidney disease. J Gen Intern Med. 2004; 19:1045-1052.9

Abbreviations: CKD, chronic kidney disease; LDL, low-density lipoprotein; HDL, high-density lipoprotein.

Although LDL is not usually elevated in patients with CKD, LDL particles tend to be smaller, denser, and more atherogenic in their form. Oxidized LDL and IDL, which are considered to be highly atherogenic, are increased. Various studies have shown increased levels of small dense LDL in non-dialysis-dependent CKD patients in comparison with the healthy controls and have indicated small dense LDL as a risk factor for CVD development. Because of the significantly modified lipid subfraction turnover, residence time of lipoproteins in the circulation is prolonged. Thus, lipoproteins are at risk of post-ribosomal modification which includes glycation, oxidation, and carbamylation. These modified lipoproteins have reduced affinity for the classic LDL receptors and are taken up by the scavenger receptors, increased in uremia, on the surface of the macrophages. High affinity for macrophages results in the accumulation of cholesterol and the formation of foam cells in the vascular walls, finally resulting in the development of accelerated atherosclerotic plaques. ${ }^{6,10,13}$

Lp (a) is an LDL-like lipoprotein which contains covalently bounded apolipoprotein (a) (apo (a)) that distinguishes it from the LDL. Apo (a) is highly homologous to plasma protease zymogene plasminogen, and it is believed that it competes with plasminogen for binding to plasminogen receptors, fibrinogen, and fibrin. All these effects lead to promoted thrombogenesis by fibrinolysis inhibition. Recent evidences have shown that $\mathrm{Lp}$ (a) is highly associated with CVD incidence in the general population. Various studies, in both healthy individuals and CKD patients, have shown strong and negative association between apo (a) isoform size and the serum Lp (a) levels. Thus, serum Lp (a) levels depend on apo (a) isoform size and are highly genetically determined by the apo (a) gene. As mentioned earlier, individuals with predominantly low molecular weight apo (a) isoforms have on average, higher Lp (a) plasma concentrations. In patients with CKD, Lp (a) concentration is as well influenced by GFR; hence, patients with large apo (a) isoforms, and not those with small isoforms, tend to have increased Lp (a) levels early in CKD stage 1, even before GFR is significantly decreased. Finally, prospective studies have found small apo (a) isoform to be an even stronger predictor of total and cardiovascular mortality in CKD patients than total Lp (a) plasma level., ${ }^{3,6,12}$

Patients with CKD have decreased HDL in comparison with individuals with preserved kidney function. This state places them at higher risk for atherosclerosis development, especially considering the results of various epidemiological studies that presented HDL as a negative risk factor for atherosclerosis. The main function of HDL is reverse cholesterol transport, a process that includes cholesterol transport from the arterial wall to the liver for further excretion. This together with HDL-mediated inhibition of inflammation, platelet adhesion, and LDL oxidation serves in normal circumstances against atherosclerosis, but in CKD patients, protective function of HDL is diminished because of several mechanisms. First, patients who have impaired kidney function often have decreased levels of apolipoproteins AI and AII, the main components of HDL. Furthermore, in CKD patients, the activity of lecithin-cholesterol acyltransferase, the enzyme important for the esterification of free cholesterol in HDL, is impaired. On the other hand, the activity of cholesterol ester transfer protein (CETP), which supports the transfer of cholesterol esters from HDL to triglyceride-rich lipoproteins, is increased. All these processes are responsible for the decreased serum level of HDL. Besides its impaired function as the cholesterol carrier, patients with CKD have reduced activity of the HDLassociated enzymes, such as paraoxonase, which may be responsible for impaired antioxidative and anti-inflammatory function of HDL. All these factors can contribute to accelerated atherogenesis in this specific population. ${ }^{6,12,13}$

\section{Dyslipidemia in nephrotic syndrome}

There is an important difference in lipid profile between patients with and without nephrotic syndrome. In nephrotic 
syndrome, lipid profile is significantly atherogenic with increased TC and LDL, which is crucial for the diagnosis of nephrotic syndrome. In patients with CKD stage 1-4 with nephrotic syndrome, hypercholesterolemia occurs because LDL production is increased and catabolism is decreased. LDL clearance is slower because of the decreased function of hepatic LDL receptors. The mechanism of this insufficiency of receptor function is not completely known; however, studies on experimental animals have shown that an ineffective translation and/or an increased LDL-receptor protein turnover could be the main processes behind these changes. Also, an inverse correlation between serum albumin levels and TC and LDL levels is presented. ${ }^{8,10,12,14}$

Besides hypercholesterolemia, hypertriglyceridemia often occurs in nephrotic syndrome, and it seems to be caused mostly by the decreased catabolism of triglycerides. Delipidation from triglyceride-rich lipoproteins, which is mediated by lipoprotein lipase and hepatic lipase, is impaired resulting in the accumulation of VLDL and remnant lipoproteins such as IDL. Impaired function of these enzymes may be caused by the loss of their activator cofactor in the urine. In addition, it seems that expression of these enzyme genes is downregulated in patients with nephrotic syndrome. ${ }^{10,12}$

HDL levels are normal or even low in patients with nephrotic range proteinuria because of the mechanism similar to one described in the pathogenesis of the CKD without proteinuria. ${ }^{12} \mathrm{CKD}$ patients with nephrotic range proteinuria have severely increased $\mathrm{Lp}$ (a) levels regardless of their apo (a) isoforms. This is probably due to heavy protein loss and consequent increased production of $\mathrm{Lp}$ (a) in the liver. ${ }^{10,12}$

\section{Dyslipidemia in patients on dialysis HD}

CKD patients who are on HD usually have similar lipid profile to those with non-dialysis-dependent CKD. TC and LDL levels are generally relatively normal, triglyceride levels are elevated, and HDL is low. In these patients, LDL is rarely markedly elevated. However, the K/DOQI guideline on dyslipidemias in CKD patients has reported that 55.7\% of patients on HD have LDL levels $>100 \mathrm{mg} / \mathrm{dL}$. These quantitative lipid abnormalities, as well as those qualitative hidden behind, play a role in atherosclerosis and cardiovascular mortality in HD patients. Approximately $50 \%$ of ESRD patients die because of CVD, and cardiovascular mortality is 30 times higher in dialysis patients. ${ }^{4,10,14}$

HD patients have moderately increased apoB and significantly increased apoC-III. Triglyceride-rich apoB containing lipoproteins (VLDL and IDL) are elevated because of decreased activities of lipoprotein lipase and hepatic lipase, resulting in hypertriglyceridemia. The same mechanism occurs in non-dialysis-dependent CKD patients. Besides this mechanism, some factors related to the process of HD itself may contribute to the increase of triglyceride levels in HD patients. It is possible that low molecular weight heparin used for anticoagulation in this patients group may potentate triglyceride elevation. It is believed that heparin releases lipoprotein lipase from the endothelia surface; hence, its prolonged use may cause depletion in lipoprotein lipase and thus reduce triglyceride-rich lipoproteins catabolism. However, controversy surrounds the association between heparin use and HD-induced dyslipidemia because some studies have reported and others have not revealed any such association. Also, it is not so clear whether the type of the membrane used in HD has any influence on serum triglyceride levels. Some studies have shown the use of high flux polysulfone or cellulose triacetate membranes to be associated with a significant reduction in serum triglyceride levels. The reason for this reduction may be due to increased apoC-II/C-III ratio, resulting in increased lipoprotein lipase activity and improvement in lipolysis of the intravascular triglyceriderich lipoproteins. ${ }^{10,12}$

HD patients also have increased plasma Lp (a), which is isoform specific. Malnutrition and inflammation are usually present in this group of patients and together with the impaired clearance of apo (a) may be responsible for these alterations..$^{12}$

In HD patients, HDL levels are reduced. A study of 183 patients treated with $\mathrm{HD}^{15}$ without cholesterol medications has shown increased plasma levels of CETP in approximately one-third of the patients, which can be one of the factors responsible for these changes. ${ }^{10}$ In addition, HDL levels may be affected by the type of membrane or dialysate that is used in HD procedure. Thus, the use of high-flux membrane in comparison with low-flux membrane can increase HDL levels. Also, the use of bicarbonate dialysate results in elevated HDL levels more often than the use of acetate dialysate. ${ }^{12}$

As mentioned earlier, behind the absence of hyperlipidemia, more atherogenic lipid profile in HD patients is usually presented. Shoji et a ${ }^{16}$ have compared 210 chronic HD patients and 223 age- and sex-matched healthy controls. The patients treated with HD had lower TC and higher triglyceride than the controls. Also, HD patients had higher VLDL and IDL but lower HDL and LDL in comparison with the controls. Despite lower mean LDL levels, HD patients had significantly decreased cholesterol/triglyceride ratio, 
reflecting the domination of more atherogenic small dense LDL. Finally, other studies have also shown that HD patients have elevated Lp (a) and oxidized LDL forms even in cases in which LDL levels are mainly normal. ${ }^{10,17}$

Various clinical trials have shown reduction of LDL levels to be associated with CVD mortality reduction in the general population. However, it is obvious that in HD patients association between dyslipidemia and CVD mortality is not that simple as in the general population. Observational studies have noted the term "reverse epidemiology" between TC levels and risk of all-cause mortality. In other words, lower TC levels are associated with a higher mortality rate. Malnutrition/inflammation could be responsible for these observations. ${ }^{9,10}$ A retrospective study of $12,000 \mathrm{HD}$ patients ${ }^{18}$ have shown that patients with low TC $(<100 \mathrm{mg} / \mathrm{dL}[2.6 \mathrm{mmol} / \mathrm{L}])$ had mortality risk more than 4 times higher in comparison with patients with TC levels. between 200 and $250 \mathrm{mg} / \mathrm{dL}$ $(5.2-6.5 \mathrm{mmol} / \mathrm{L})$. The association between TC and mortality formed a U-shaped curve that seemed more linear after adjustment for serum albumin. ${ }^{9,10}$

One 10-year prospective study followed 1,167 Japanese HD patients..$^{19}$ This study has shown low TC to be independently associated with higher C-reactive protein (CRP) and mortality in patients with low albumin. ${ }^{10}$ Another prospective study followed 823 dialyzed patients for a median of 2.4 years and classified them due to presence or absence of inflammation and/or malnutrition at baseline. Inflammation and/or malnutrition were defined by albumin serum levels, CRP or interleukin-6. An increase in baseline TC of $1 \mathrm{mmol} / \mathrm{L}$ was associated with a reduction of all-cause mortality in the presence of inflammation/malnutrition. ${ }^{10}$

These studies results reveal that low serum TC levels are associated with increased mortality in HD patients, suggesting hypocholesterolemia as a surrogate for malnutrition or inflammation. ${ }^{9,10}$

\section{PD}

Patients on PD have some form of "uremic dyslipidemia" just like those on HD, but their lipid profile is more atherogenic in nature with more altered dyslipidemia when compared with those on HD., ${ }^{3,10,12}$ PD patients show increased levels of triglyceride, apoB containing VLDL and IDL, TC and LDL, as well as small dense LDL and Lp (a) while HDL levels are low. These differences may be attributed to PD per se, but may also be associated with the selection of dialytic modality. One crosssectional study compared 31 patients on continuous ambulatory peritoneal dialysis (CAPD), 30 patients treated with $\mathrm{HD}$, and 27 healthy controls. Patients on CAPD had significantly higher mean TC (6.8 vs $5.1 \mathrm{mmol} / \mathrm{L}, p<0.001)$, LDL (4.6 vs $3.2 \mathrm{mmol} / \mathrm{L}, p<0.001$ ), VLDL ( 1 vs $0.7 \mathrm{mmol} / \mathrm{L}, p<0.05$ ), and triglyceride ( 2.3 vs $1.5 \mathrm{mmol} / \mathrm{L}, p<0.01)$ than patients on $\mathrm{HD}$. Also, there was no significant difference in HDL (1.1 vs 1.3 $\mathrm{mmol} / \mathrm{L}$ in $\mathrm{HD}, p=\mathrm{NS}$ ) in patients on CAPD. ${ }^{10}$

Hypertriglyceridemia is predominantly present in patients on PD. The exact mechanism is not completely understood, but it is believed that a significant absorption of glucose from the dialysis fluid may induce hepatic lipoprotein synthesis or may cause an increase in insulin levels resulting in the enhanced hepatic VLDL synthesis and secretion. Recent studies have shown an improvement in lipid profile when the overnight dwell has been switched from a dextrose-based solution to icodextrin, because of the reduced glucose overload. ${ }^{10,12}$

Higher TC, LDL and small dense LDL can be explained by several mechanisms similar to those in nephrotic syndrome and those related to the protein loss. In PD patients, great amounts of plasma proteins are lost into the peritoneal dialysate, which stimulates the liver to produce albumin and other proteins, including cholesterol-enriched lipoproteins in an attempt to compensate for the protein loss. Also, various lipoproteins, such as HDL, are lost through the peritoneal cavity which may contribute to its decreased levels. ${ }^{10,12}$

Compared to HD, patients on PD have increased Lp (a) plasma levels which are not isoform specific. Also, Lp (a) levels are usually more elevated in patients on PD than those on HD. ${ }^{10}$ This may be due to enhanced Lp (a) synthesis in the liver as a result of the increased protein loss. ${ }^{12}$ In their large multicenter cross-sectional study, Kronenberg et $\mathrm{a}^{17}$ have shown that $34 \%$ of HD patients and $42 \%$ of CAPD patients had serum Lp (a) greater than the 75th percentile of the healthy control group $(>0.92 \mathrm{mmol} / \mathrm{L}, p<0.005$ for HD vs PD). ${ }^{10}$

\section{Dyslipidemia in renal transplant patients}

CVD is the most common cause of death in the renal transplant population. These patients often die with a functional graft because of CVD, and thus, CVD is the most common cause of graft loss. Dyslipidemia is an important risk factor for CVD development, and its incidence in the 1st year following kidney transplantation can go beyond $50 \%$. Lipid profile in renal transplant patients includes elevated plasma levels of TC, LDL, VLDL, and triglyceride while HDL is significantly reduced. Many factors can influence post-transplant dyslipidemia including age, genetic predisposition, obesity and reduced physical activity, nephrotic syndrome, hypothyreosis, diabetes mellitus, chronic liver disease, and immunosuppressive agents. These medications 
include calcineurin inhibitors (CNIs), primarily cyclosporine, followed by corticosteroids and mTOR inhibitors. ${ }^{3,21-23}$ Corticosteroids can induce dyslipidemia by the stimulation of liver lipid synthesis, especially VLDL synthesis, as well as by decreasing LDL hepatic intake. Cyclosporine is responsible for the elevation of TC and LDL and reduction of HDL levels which is dose dependent. These changes in the lipid profile are caused by the binding of cyclosporine to hepatic LDL receptors and consequently disrupted liver LDL uptake. Tacrolimus, azathioprine, and mycophenolate mofetil usually cause minimal changes in the lipid profile; hence, the replacement of cyclosporine with one of these medications can improve serum triglyceride and cholesterol levels, but does not have any effect on HDL levels. mTOR inhibitor sirolimus significantly increases triglyceride plasma levels by inducing the synthesis and secretion of liver triglyceride and VLDL. When used in combination, sirolimus and cyclosporine synergistically contribute to the development of dyslipidemia in kidney transplant recipients. Finally, dyslipidemia in kidney transplant patients can have negative impact on kidney graft function which leads to the conclusion that lipid lowering could have beneficial effect on the graft function. , $^{3,21}$

One recent retrospective case-control and before-after study has shown an improvement in the lipid profile after kidney transplantation when compared with ESRD. In this study, a retrospective case-control analysis of 816 patients admitted to the Royal Hobart Hospital in 2008-2009 with different degrees of kidney impairment and retrospective before-after cohort analysis of 60 patients who received a transplanted kidney between 1999 and 2009 was performed. After kidney transplantation, HDL has significantly increased $(p<0.0001)$ and triglyceride levels have significantly decreased ( $p=0.007)$. TC and LDL levels haven't significantly changed. HDL and triglycerides level improvement following kidney transplantation was associated with successful engraftment and maintenance of graft function. If graft function was not successful, HDL again decreased and triglycerides levels increased. Some limitations of this study have to be considered, including immunosuppressive medications which could affect lipoprotein metabolism and concentration as well as a frequent statin use in the studied population which could affect TC and LDL levels after kidney transplantation. But according to these results, it can be concluded that kidney function is an important determinant of HDL and triglyceride concentrations in CKD and kidney transplant patients which can affect CVD risk associated with $\mathrm{CKD},{ }^{24}$

\section{Management of dyslipidemia}

From the previously described pathogenesis of dyslipidemia in patients with renal impairment, it is obvious that dyslipidemia accelerates atherogenic processes and increases CVD risk in this population. However, dyslipidemia can also worsen the decline in the kidney function because of deposition of lipids in the kidney. In 1982, Moorhead et $\mathrm{al}^{25}$ investigated the association between hyperlipidemia and renal function impairment, and since then, various new evidences have supported this hypothesis. It is believed that deposition of lipid may cause damage to mesangial cells, endothelial cells, and glomerular podocytes by inducing inflammatory reaction and matrix production in glomerular mesangium, resulting in glomerulosclerosis. Lipid accumulation may, with time, increase loss of functioning of nephrons, resulting in worsening of the renal function. ${ }^{23}$

According to the potential negative effects of dyslipidemia on CVD development and impairment of renal function, it is logical to speculate that initiation of dyslipidemia controlling therapy in patients with renal impairment could mitigate its negative renal and cardiovascular consequences. However, evidences of these beneficial effects are still lacking mostly because patients with CKD were often excluded from major trials that evaluated dyslipidemia treatment and its impact on CVD prevention. Clinical trials have confirmed that reduction of LDL leads to decreased cardiovascular mortality, but in the CKD population, this association is less clear. Some authors claim that dyslipidemia associated with renal impairment should be considered as a coronary heart disease-risk equivalent similar to diabetes mellitus and thus should be intensively treated. ${ }^{23,26}$

\section{Management of dyslipidemia in CKD $(\mathrm{GI}-4)$}

Statins are medications that are very often prescribed for lipid lowering in the general population, and various large, randomized, prospective studies have shown that their use leads to significant decrease in cardiovascular events. ${ }^{6,12,23} \mathrm{In}$ the uremic patients, statins are as effective in lowering the TC and LDL as they are in the general population, but their beneficial effect on cardiovascular morbidity and mortality in CKD patients seems to depend on the severity of renal dysfunction. Also, it should be mentioned that statins do not have an effect on hyperglyceridemia, elevated Lp (a) and decreased HDL levels which are often presented in CKD patients. ${ }^{12,23}$ Various studies have shown statins to be effective in the cardiovascular risk reduction. Some of these studies are the Treatment to New Targets (TNT), Heart Protection 
Study (HPS), combined analysis of the Cholesterol and Recurrent Events (CARE), West of Scotland Coronary Prevention Study, and Long-term Intervention with Pravastatin in Ischemic Disease studies. ${ }^{23}$

The HPS included 20,000 British men and women aged 40-80 years who had an increased risk of CVD death because of diabetes, CVD, and other atherosclerotic diseases. The study was conducted for 5 years. The primary outcomes have been to evaluate total mortality, fatal and nonfatal vascular events after cholesterol lowering with $40 \mathrm{mg}$ /day simvastatin. In this study, a subgroup of $1,329 \mathrm{CKD}$ patients whose creatinine ranged from 1.3 to $2.3 \mathrm{mg} / \mathrm{dL}$ have been enrolled. In this subgroup, relative risk (RR) reduction was $28 \%(95 \%$ confidence interval $[\mathrm{CI}] 0.72-0.85 ; p<0.05)$. Compared with the control group, the event rate has been reduced in the group that was receiving simvastatin (control group 39.2\% vs simvastatin group $28.2 \%$ ) and has resulted in the $11 \%$ absolute risk reduction. ${ }^{27}$ Similar results have been obtained in the Anglo-Scandinavian Cardiac Outcomes Trial in which a subgroup of 6,517 patients with kidney dysfunction have been analyzed after receiving $10 \mathrm{mg}$ of atorvastatin per day during a median of 3.3 years follow-up. Atorvastatin has significantly reduced the risk for the primary composite end point of nonfatal myocardial infarction (MI) and fatal coronary heart disease by $39 \% .^{12}$

In the CARE study, 4,159 patients with hyperlipidemia and history of acute MI were randomized to receive pravastatin or placebo. After a 5-year follow-up period, their cardiovascular morbidity and mortality have been evaluated. In the subgroup of 1,700 patients with mild CKD (creatinine clearance $<75$ $\mathrm{mL} / \mathrm{min}$ ) receiving $40 \mathrm{mg}$ pravastatin per day, there was a $28 \%$ (95\% CI 0.55-0.95; $p=0.02$ ) RR reduction and a $4 \%$ absolute risk reduction in the primary end point that included death from coronary disease and symptomatic nonfatal MI. ${ }^{23,27}$

Some studies have shown statins to be renoprotective not just because of their ability to reduce lipid levels, but because of their ability to reduce interstitial inflammation, improve renal hemodynamics and decrease glomerular proteinuria which could result in the reduction of renal function decline. This was confirmed by the analysis of a subgroup of patients in the TNT study. Patients who have been receiving $80 \mathrm{mg}$ of atorvastatin were compared with patients who have been receiving $10 \mathrm{mg}$ of atorvastatin per day after a 5-year followup period. An increase in GFR has been observed in both the groups of patients, but the increase was higher in the 80 mg receiving group..$^{13,23}$

According to the Kidney Disease: Improving Global Outcomes (KDIGO) guidelines in adults with newly identified
CKD, evaluation of a lipid profile (TC, LDL, HDL, and triglycerides) should be performed mainly because of the potential severe hypercholesterolemia or hypertriglyceridemia diagnosis and potential secondary cause establishment. There is no precise evidence on usefulness of the measurement of the lipid status and its potential to improve clinical outcomes, but the levels of triglyceride $>11.3 \mathrm{mmol} / \mathrm{L}$ or LDL $>4.9 \mathrm{mmol} / \mathrm{L}$ may require further evaluation. Thus, follow-up measurement of lipid levels in adults with CKD is not required for the majority of patients especially because clinical benefits of statin treatment are proportional to the baseline coronary risk rather than baseline LDL. ${ }^{28}$

Available evidence suggests absolute risk for coronary events as a deciding factor for receiving cholesterol-lowering medication in CKD patients instead of LDL levels measurement. The size of the excess risk, which is associated with increased LDL levels, decreases when eGFR levels are low in non-dialysis dependent CKD patients. ${ }^{28}$

KDIGO guidelines recommend treatment with a statin or statin/ezetimibe combination in adults aged $\geq 50$ years with eGFR $<60 \mathrm{~mL} / \mathrm{min} / 1.73 \mathrm{~m}^{2}$ who are not treated with chronic dialysis or kidney transplantation (GFR categories G3a-G5). ${ }^{28}$

The Study of Heart and Renal Protection (SHARP), international double-blind randomized trial included 9,270 CKD patients to receive simvastatin $20 \mathrm{mg}$ plus ezetimibe 10 mg daily or placebo during the mean 4.9 years of follow-up period. Most non-dialysis-dependent CKD patients $(n=6,247)$ were in stages 3 and 4 (mean eGFR $=27 \mathrm{~mL} / \mathrm{min} / 1.73 \mathrm{~m}^{2}$ ), and the remaining 3,023 patients were receiving dialysis (HD and PD). Combination of the simvastatin plus ezetimibe has led to the $0.83 \mathrm{mmol} / \mathrm{L}$ mean reduction in $\mathrm{LDL}$ in the treatment group in comparison with the placebo. Combination therapy caused significant $17 \% \mathrm{RR}$ reduction of the primary outcome of major atherosclerotic events (coronary death, MI, nonhemorrhagic stroke, or any revascularization) compared with placebo (HR 0.83; 95\% CI 0.74-0.94). However, the effect of treatment on coronary events (coronary heart disease death and nonfatal MI) was not different $(p=0.37)$. In the 6,247 non-dialysis-dependent CKD patients, simvastatin/ezetimibe therapy did not reduce the risk of progression to the ESRD. In the subgroup of dialysis-dependent patients treatment with simvastatin/ezetimibe combination did not significantly reduce the risk of the primary outcome..$^{3,26,28}$

According to SHARP results, post hoc analyses of randomized trials from the general population, as well as those focusing on the subgroups with $\mathrm{CKD}$, and KDIGO guidelines recommend such treatment in the previously 
described patients with CKD because of their generally high cardiovascular risk. ${ }^{28}$

Finally, according to KDIGO guidelines, in adults aged 18-49 years with CKD but not treated with chronic dialysis or kidney transplantation, statin treatment is recommended in the case of known coronary disease (MI or coronary revascularization), diabetes mellitus, prior ischemic stroke or estimated 10-year incidence of coronary death or nonfatal $\mathrm{MI}>10 \%{ }^{28}$

In adults with CKD (including those treated with chronic dialysis or kidney transplantation), according to KDIGO guidelines, therapeutic lifestyle changes should be advised. Therapeutic lifestyle changes include dietary modification, weight reduction, increased physical activity, reducing alcohol intake, and treatment of hyperglycemia if present. Evidence shows that lifestyle changes can reduce serum triglyceride levels in CKD patients is weak but may improve general health. However, according to KDIGO guidelines, CKD patients with high fasting triglyceride serum levels $(5.65 \mathrm{mmol} / \mathrm{L}$ or $500 \mathrm{mg} / \mathrm{dL})$ should adopt a low-fat diet ( $<15 \%$ total calories), reduce monosaccharide and disaccharide intake as well as the total amount of dietary carbohydrates, and use fish oils to replace some long-chain triglycerides. Dietary changes should be performed very carefully in malnourished patients..$^{28}$

Because of hypertriglyceridemia being presented in CKD patients, fibrates seem to be the logical treatment option for these patients. However, evidence from the randomized controlled trials about potential protective effect of these medications is still lacking. Furthermore, these medications are not often used in practice because of the concern of rhabdomyolysis, worsening of the renal function, impairment of the liver function or development of elevation of homocysteine in CKD patients. ${ }^{23} \mathrm{KDIGO}$ guidelines recommend fibric acid derivate to be used only in rare cases of markedly elevated fasting levels of serum triglycerides $(11.3 \mathrm{mmol} / \mathrm{L}$ or 1,000 $\mathrm{mg} / \mathrm{dL}$ ). In such cases, doses of fibric acid derivate have to be adjusted for kidney function and should not be used with statins because of potential toxicity. Taken together, fibric acid derivates are not recommended for prevention of pancreatitis or reduce cardiovascular risk in CKD patients with hypertriglyceridemia. ${ }^{28}$

\section{Management of dyslipidemia in nephrotic syndrome}

Nephrotic-range proteinuria is usually associated with hypercholesterolemia and hypertriglyceridemia. It is possible that reduction in proteinuria could improve dyslipidemia in these patients. However, evidence confirming this hypothesis is inconsistent. Only a few randomized controlled trials have demonstrated the association between therapies that can reduce the excretion of urine protein and beneficial effect on the lipid profile. Angiotensin II-converting enzyme inhibitors (ACE-I), angiotensin II receptor blockers (ARBs), and diets with low protein intake often reduce the excretion of urine protein. One randomized controlled trial included 17 nephrotic patients treated with an ACE-I. After the followup period, the reduction in the excretion of urine protein and mean TC have been observed when compared with the placebo group. Evidence of the effect of low-protein diets on the lipid profile in nephrotic-range proteinuria patients is also lacking. Modification of Diet in Renal Disease study has reported a tendency of serum TC and LDL levels lowering with the reduction of dietary protein intake. ${ }^{29}$

It is well known that ACE-I and ARBs can decline the progression of the renal disease in patients with and without proteinuria. Thus, patients with CKD and proteinuria should be treated with ACE-I or ARBS despite the levels of plasma lipids. ${ }^{29}$

The majority of CKD patients with eGFR $\geq 60 \mathrm{~mL} /$ $\mathrm{min} / 1.73 \mathrm{~m}^{2}$ have proteinuria. ${ }^{28}$ These patients are rarely recognized in the randomized trials of statins. According to the current evidence, the presence of albuminuria does not decrease the beneficial effects of the statin treatment. The CARD and CARE trials have not found any significant interaction between the presence of albuminuria and statin treatment effect on cardiovascular events. Considering the high cardiovascular risk in the CKD stages 1 and 2 patients and the evidence demonstrating statins to be equally effective in the presence or absence of proteinuria, KDIGO guidelines recommend that adults aged $\geq 50$ years with $\mathrm{CKD}$ and $\mathrm{eGFR} \geq 60 \mathrm{~mL} / \mathrm{min} / 1.73$ $\mathrm{m}^{2}$ (GFR categories G1-G2) should be treated with a statin. ${ }^{28}$

\section{Management of dyslipidemia in patients on dialysis $\mathrm{HD}$ and PD}

It is well known that HD and PD patients are at high risk of CVD morbidity and mortality, but data about the association of lipid-lowering treatment and reduction of cardiovascular mortality in this population is conflicting. The majority of $\mathrm{CV}$ deaths in dialysis patients are caused by sudden death that may be because of electrolyte abnormalities or arrhythmia or cardiomyopathy that may be due to chronic extracellular fluid volume overload and not by the atherosclerotic coronary heart disease. Thus, it is possible that positive effects of statins on the atherosclerotic events may have been diluted. Some epidemiological studies have shown statins to be effective in cardiovascular mortality reduction. US Renal Data System 
Dialysis Morbidity and Mortality Study included 3,700 HD patients who were followed for 2 years. In the group of patients receiving statins, RR reduction in total mortality was $32 \%$ in comparison with the group of patients receiving fibrates that did not develop any reduction in cardiovascular or total mortality. Similar positive effects of statins on the relative reduction in total mortality have been shown in the Dialysis Outcomes Practice Patterns observational study. However, the prospective, randomized trials that have evaluated the effects of the statin use in this patient population have not shown such positive results as epidemiological studies; their results seem to be disappointing. ${ }^{10,12,27}$

The 4D Study (Die Deutsche Diabetes Dialyse Studie) is a multicenter, double-blind, randomized trial that included 1,255 patients with type 2 diabetes treated with HD. They have been receiving maintenance HD for $<2$ years. Patients were randomized to receive atorvastatin $20 \mathrm{mg}$ /day or placebo and followed up for 4 years. After 4 weeks, atorvastatin treatment reduced the LDL levels by $42 \%$ in comparison with $1.3 \%$ reduction in the placebo group. Despite this significant reduction in LDL levels, atorvastatin had a non-significant 8\% $\mathrm{RR}$ reduction $(95 \%$ CI $0.77-1.10 ; p=0.37$ ) on the combined primary end point (a composite of cardiac death, nonfatal MI, and stroke). Moreover, atorvastatin increased the risk of fatal stoke in this patient population (RR 2.03; 95\% CI 1.05-3.93; $p=0.04)$. The secondary endpoint of combined cardiac events was significantly decreased (RR 0.82 ; 95\% CI 0.68-0.99; $p=0.03$ ). However, not all combined cerebrovascular events and total mortality was significantly reduced. , $^{6,13,26-28}$

The A Study to Evaluate the Use of Rosuvastatin in Subjects on Regular Dialysis: An Assessment of Survival and Cardiovascular Events (AURORA) is an international prospective double-blind study that included 2,776 HD patients. They have been randomized to receive $10 \mathrm{mg}$ of rosuvastatin per day or placebo. The primary combined end points were nonfatal MI, nonfatal stroke, and cardiovascular death. After a period of 3 months, the reduction of LDL was $43 \%$ in the rosuvastatin group. Despite this significant reduction in LDL levels, the combined primary endpoint was not reduced after a median follow-up period of 3.8 years (HR 0.96; 95\% CI 0.84-1.11; $p=0.59)$. Furthermore, there was no reduction in the risk of the individual components of the primary endpoint or reduction in all-cause mortality ((HR 0.96; 95\% CI 0.86-1.07; $p=0.51)$. The results of the AURORA study may lead to the concept of "a point of no return". In other words, there might be a point in the impairment of the renal function in which beneficial effects of a statin therapy are modified by the uremic environment. Despite some of the limitations of these studies, as well as the previously described SHARP study, their results have caused a great amount of concerns about efficiency of the statin use in the dialyzed population. ${ }^{12,26,28}$

Although dialyzed patients have high CVD risk, these data suggest that clinical benefits of statin or statin/ezetimibe treatment are uncertain. Thus, KDIGO guidelines do not recommend an initiation of statin or statin/ezetimibe treatment in most adult, dialysis-dependent CKD patients. Still, patients can use statins with the awareness of a relatively small and uncertain CVD risk reduction. In patients who are already receiving statin or statin/ezetimibe therapy at the time of dialysis initiation, according to KDIGO guidelines such therapy should be continued with a regular monitoring of the patients' condition given the lack of evidence that could advise different approach. ${ }^{28}$

\section{Management of dyslipidemia in renal transplant patients}

Renal transplant recipients are considered to be at high risk for CVD development. Managing dyslipidemia through the lifestyle changes is often insufficient; hence, medications are often needed. Statins are the most commonly used medications in this patient group and are proved to be safe and effective, not only in the lipid profile improvement, but also in protecting graft function. ${ }^{22}$

The Assessment of Lescol in Renal Transplantation (ALERT) is a large, randomized, placebo-controlled trial designed to evaluate the effect of statin treatment on the cardiovascular risk reduction. The study included 2,102 renal transplant recipients aged 30-75 years with functional allograft who were randomized to receive $40-80 \mathrm{mg} /$ day fluvastatin therapy or placebo and followed up for 5-6 years. The fluvastatin therapy led to nonsignificant (17\%) reduction in the primary outcome of coronary death or non-fatal MI in comparison to placebo (RR 0.83; 95\% CI 0.64-1.06). However, the RR reduction in cardiac death or definite nonfatal MI caused by fluvastatin therapy was significant (35\%) compared with placebo (HR 0.65; 95\% CI 0.48-0.88). Importantly, unblended extension study showed significant decrease in the original primary outcome due to fluvastatin therapy after 6.7 years of follow-up. The lipid-lowering and cardiovascular benefits of fluvastatin therapy observed from the ALERT study are comparable with the beneficial effects of statin therapy in the general population. Thus, KDIGO guidelines suggest statin treatment in adult kidney transplant recipients. ${ }^{9,13,14,26,28}$

When used in combination, cyclosporine can interact with some of the statins resulting in potential myopathy and rhabdomyolysis. Thus, in renal transplant recipients receiving a cyclosporine, a lower dose of statin should be used with more careful monitoring of these patients. ${ }^{14,22}$ 
Finally, various studies have shown ezetimibe to be effective when used with statins. In their retrospective review, Buchanan et a ${ }^{30}$ evaluated the safety and efficiency of ezetimibe in renal transplant patients. Monotherapy or combination therapy resulted in a mean reduction in TC of $23.3 \%$, triglycerides $40.2 \%$, LDL $16.8 \%$, and HDL $4.8 \%$ after 3.1 months of therapy. According to these results, ezetimibe is safe and effective for dyslipidemia treatment in renal transplant recipients, both as a combination or monotherapy. Also, ezetimibe does not change the CNI levels and renal function. ${ }^{30}$ Similar results have been observed by the study of Türk et al. ${ }^{31}$

\section{Conclusion}

Dyslipidemia is often present in patients with renal impairment and differs quantitatively and qualitatively in nondialyses-dependent patients, patients with nephrotic range proteinuria, ESRD patients, and renal transplant recipients. It can affect kidney function and significantly increase the risk of CVD development. Thus, diagnosis and management of these patients are important to potentially improve their clinical outcome. However, according to recent large clinical trials, effects of treatment in reducing CVD morbidity and mortality in patients with renal impairment are not as promising as in general population. Thus, further research is needed to find the most beneficial treatment options for these patients.

\section{Disclosure}

The authors report no conflicts of interest in this work.

\section{References}

1. Parmar JA, Joshi AG, Chakrabarti M. Dyslipidemia and chronic kidney disease. ISRJ. 2014;3:396-397.

2. Charles RH, Terry AJ. Managing dyslipidemia in chronic kidney disease. J Am Coll Cardiol. 2008;51:2375-2384.

3. Ahmed MH, Khalil AA. Ezetimibe as a potential treatment for dyslipidemia associated with chronic renal failure and renal transplant. Saudi J Kidney Dis Transplant. 2010;21:1021-1029.

4. Balode AA, Khan ZH. Serum lipid profile in chronic kidney disease patients on haemodialysis. IJAR. 2013;3:20-22.

5. Burmeister JE, Mosmann CB, Veridiana Borges Costa VB, et al. Prevalence of cardiovascular risk factors in hemodialysis patients - the CORDIAL study. Arq Bras Cardiol. 2014;102:473-480.

6. Kwan BCH, Kronenberg F, Beddhu S, Cheung AK. Lipoprotein Metabolism and lipid management in chronic kidney disease. JAm Soc Nephrol. 2007;18:1246-1261.

7. Chen S-C, Hung C-C, Kuo M-C, et al. Association of dyslipidemia with renal outcomes in chronic kidney disease. PLoS One. 2013;8:e55643.

8. Cases A, Coll E. Dyslipidemia and the progression of renal disease in chronic renal failure patients. Kidney Int Suppl. 2005;(99):S87-S93.

9. Weiner DE, Sarnak MJ. Managing dyslipidemia in chronic kidney disease. J Gen Intern Med. 2004;19:1045-1052.

10. Shurraw S, Tonelli M. Statins for treatment of dyslipidemia in chronic kidney disease. Perit Dial Int. 2006;26:523-539.
11. Wanner C, Ritz E. Reducing lipids for CV protection in CKD patientscurrent evidence. Kidney Int. 2008;74:24-28.

12. Tsimihodimos V, Mitrogianni Z, Elisaf M. Dyslipidemia associated with chronic kidney disease. Open Cardiovasc Med J. 2011;5:41-48.

13. Piecha G, Adamczak M, Ritz E. Dyslipidemia in chronic kidney disease. Pol Arch Med Wewn. 2009;119:487-492.

14. Molitch ME. Management of dyslipidemias in patients with diabetes and chronic kidney disease. Clin JAm Soc Nephrol. 2006;1:1090-1099.

15. Kimura H, Miyazaki R, Imura T, Masunaga S, Suzuki S, Gejyo F, Yoshida $\mathrm{H}$. Hepatic lipase mutation may reduce vascular disease prevalence in hemodialysis patients with high CETP levels. Kidney Int. 2003;64:1829-1837.

16. Shoji T, Nishizawa Y, Kawagishi T, et al. Atherogenic lipoprotein changes in the absence of hyperlipidemia in patients with chronic renal failure treated by hemodialysis. Atherosclerosis. 1997;131:229-236.

17. Kronenberg F, Lingenhel A, Neyer U, et al. Prevalence of dyslipidemic risk factors in hemodialysis and CAPD patients. Kidney Int Suppl. 2003;84:113-116

18. Lowrie EG, Lew NL. Death risk in hemodialysis patients: the predictive value of commonly measured variables and an evaluation of death rate differences between facilities. Am J Kidney Dis. 1990;15:458-482.

19. Iseki K, Yamazato M, Tozawa M, Takishita S. Hypocholesterolemia is a significant predictor of death in a cohort of chronic hemodialysis patients. Kidney Int. 2002;61:1887-1893.

20. Liu Y, Coresh J, Eustace JA, et al. Association between cholesterol level and mortality in dialysis patients: role of inflammation and malnutrition. JAMA. 2004;291:451-459.

21. Mikolašević I, Lukenda V, Sladoje-Martinović B, et al. Metabolic complications after solid organ transplantation. Medicina Fluminensis. 2014;50:61-66.

22. Kahwaji JM, Dudek RR. How can we manage hyperlipidemia and avoid rhabdomyolysis in transplant patients? Perm J. 2006;10:26-28.

23. Mesquita J, Varela A, Medina JL. Dyslipidemia in renal disease: causes, consequences and treatment. Endocrinol Nutr. 2010;57:440-448.

24. Thompson M, Ray U, Yu R. Kidney function as a determinant of HDL and triglyceride concentrations in the Australian population. J Clin Med. 2016;5:35.

25. Moorhead JF, Chan MK, El-Nahas M, et al. Lipid nephrotoxicity in chronic progressive glomerular and tubulo-interstitial disease. Lancet. 1982;2:1309-1311.

26. Omran J, Al-Dadah A, Dellsperger KC. Dyslipidemia in patients with chronic and end-stage kidney disease. Cardiorenal Med. 2013;3: 165-177.

27. Harper CR, Jacobson TA. Managing dyslipidemia in chronic kidney disease. J Am Coll Cardiol. 2008;51:2375-2384.

28. Wanner C, Tonelli M; Kidney Disease: Improving Global Outcomes Lipid Guideline Development Work Group Members. KDIGO clinical practice guideline for lipid management in chronic kidney disease. Kidney Int Suppl. 2013;3:259-305.

29. Kidney Disease Outcomes Quality Initiative (K/DOQI) Group. K/DOQI clinical practice guidelines for managing dyslipidemia in chronic kidney disease. Am J Kidney Dis. 2003;41:1-237.

30. Buchanan C, Smith L, Corbett J, Nelson E, Shihab F. A retrospective analysis of ezetimibe treatment in renal transplant recipients. $\mathrm{Am} \mathrm{J}$ Transplant. 2006;6:770-774.

31. Türk TR, Voropaeva E, Kohnle M, et al. Ezetimibe treatment in hypercholesterolaemic kidney transplant patients is safe and effective and reduces the decline of renal allograft function: a pilot study. Nephrol Dial Transplant. 2008;23:369-373.

32. No Authors listed. Report of the National Cholesterol Education Program Expert Panel on Detection, Evaluation, and Treatment of High Blood Cholesterol in Adults. Arch Intern Med. 1988;148(1):36-69.

33. Schaefer EJ, Lamon-Fava S, Cohn SD, et al. Effects of age, gender, and menopausal status on plasma low density lipoprotein cholesterol and apolipoprotein B levels in the Framingham Offspring Study. J Lipid Res. 1994;35:779-792.

34. Kasiske BL. Hyperlipidemia in patients with chronic renal disease. $A m$ J Kidney Dis. 1998;32:142-156. 
The International Journal of Nephrology and Renovascular Disease is an international, peer-reviewed open access journal focusing on the pathophysiology of the kidney and vascular supply. Epidemiology, screening, diagnosis, and treatment interventions are covered as well as basic science, biochemical and immunological studies. The manuscript management system is completely online and includes a very quick and fair peer-review system, which is all easy to use. Visit http://www. dovepress.com/testimonials.php to read real quotes from published authors.

Submit your manuscript here: https://www.dovepress.com/international-journal-of-nephrology-and-renovascular-disease-journal 